\title{
From Galaxyzoo to dark matter
}

\author{
Carlo Artemi \\ Scientific High school " Ettore Majorana “ \\ Via dei tigli 2305018 Orvieto ( TR ), Italy \\ E-mail: carlo.artemi@poste.it
}

Studies of dark matter particles are done, or by attempt to produce it ( i.e. by LHC ) or by searching indirect signs of it ( let think to Pamela signal for possible decay of dark matter particles in high energy electrons ). In this paper it's showed a new way to study dark matter and dark energy too. The paper starts with a description of the citizens science project Galaxyzoo with an hint to its huge scientific results. In this project low-middle redshift galaxies of SSDS database are classified from point of view of their morphology. After the description it's showed as to widen this study to high or very high redshift galaxies databases. Done it will be possible to compare galaxies cataloguing in the real universe with the features of galaxies in virtual universes produced by supercomputer simulations and, because of simulations start from hypotheses on the features of dark matter and dark energy, it will be possible to determine the features themselves. Final consideration will be done on rising impact of citizens science also in fields until now reserved to professional scientists 


\section{Galaxyzoo project}

This project is a citizens science project and its aim is to classify, in a more or less detailed way, galaxies from the morphological point of view. The structure of this project is showed in the following picture

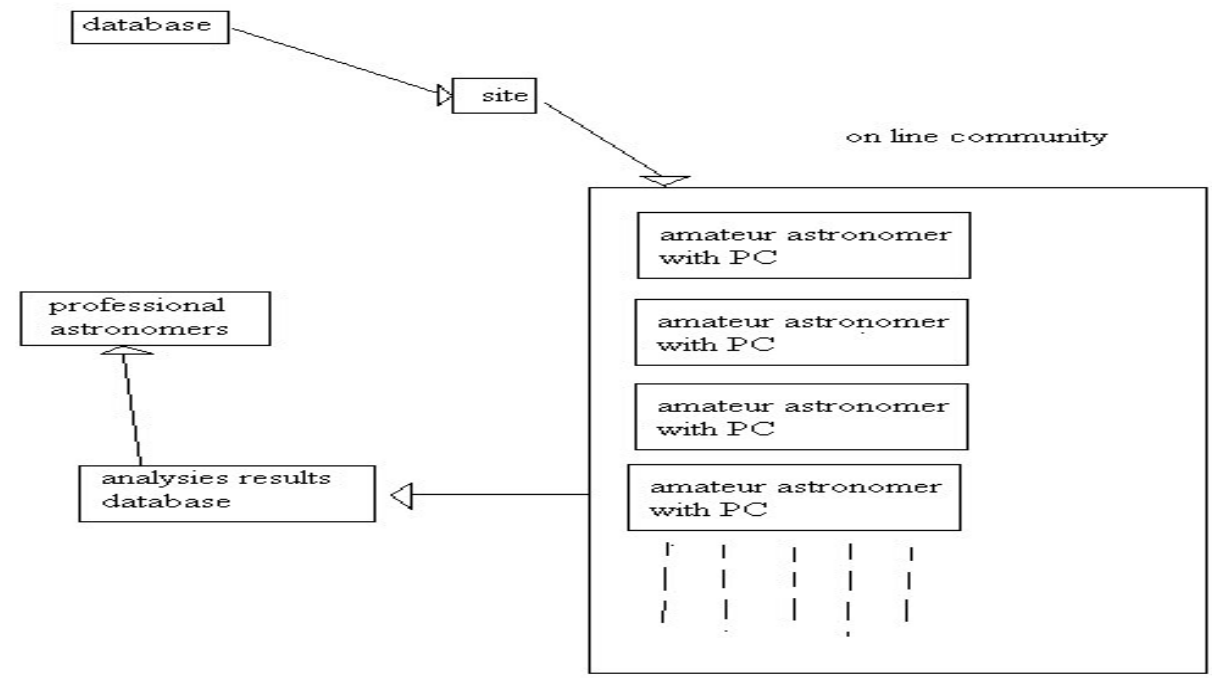

Fig. 1 A schematic vision of " modus operandi "of Galaxyzoo project

Author is one of amateur astronomers even he has made and makes researches . The project site is www.galaxyzoo.org and in this site every information on project ( from content of papers to names of professional astrophysics involved ) can be found .

The amateur astronomers register themselves, obtaining an username and a password, after they can catalogue galaxies answering to a series of multiple choices questions . Until today online community includes almost 150000 people of almost 40 countries . Scientific results has been huge. The collaboration has 17 papers published or accepted in press in high impact factor journals ( first published paper in summer 2008 ). And besides there are conference communications and citations both from specialized reviews and from newspapers as New York Times , Herald Tribune and many other among which Italian Corriere della Sera . It's practically impossible to summarize these results in few words. To give an idea of these results let cite

The discovery and the study of new objects as green galaxies ( " peas " ) ${ }^{1}$, Hanny Vooowerps ${ }^{2}$ ( quasar light echoes ?) blue elliptical galaxies ${ }^{3}$ ( the actual theories of galaxies evolutionion predict they would be red ) red spiral galaxies ${ }^{4}$ and so on

The detailed studies on relations there are among several features of Galaxies ${ }^{5},{ }^{6}$, , and in particular studies on $\mathrm{AGN}^{8}$, 
This project has grown in three stages :

1 ) Galaxyzoo 1 in which Galaxies from SSDS database ( 900000 low-middle redshift objects ) were analysed with a not.much detailed classification. An example of Galaxyzoo 1 only question is the following

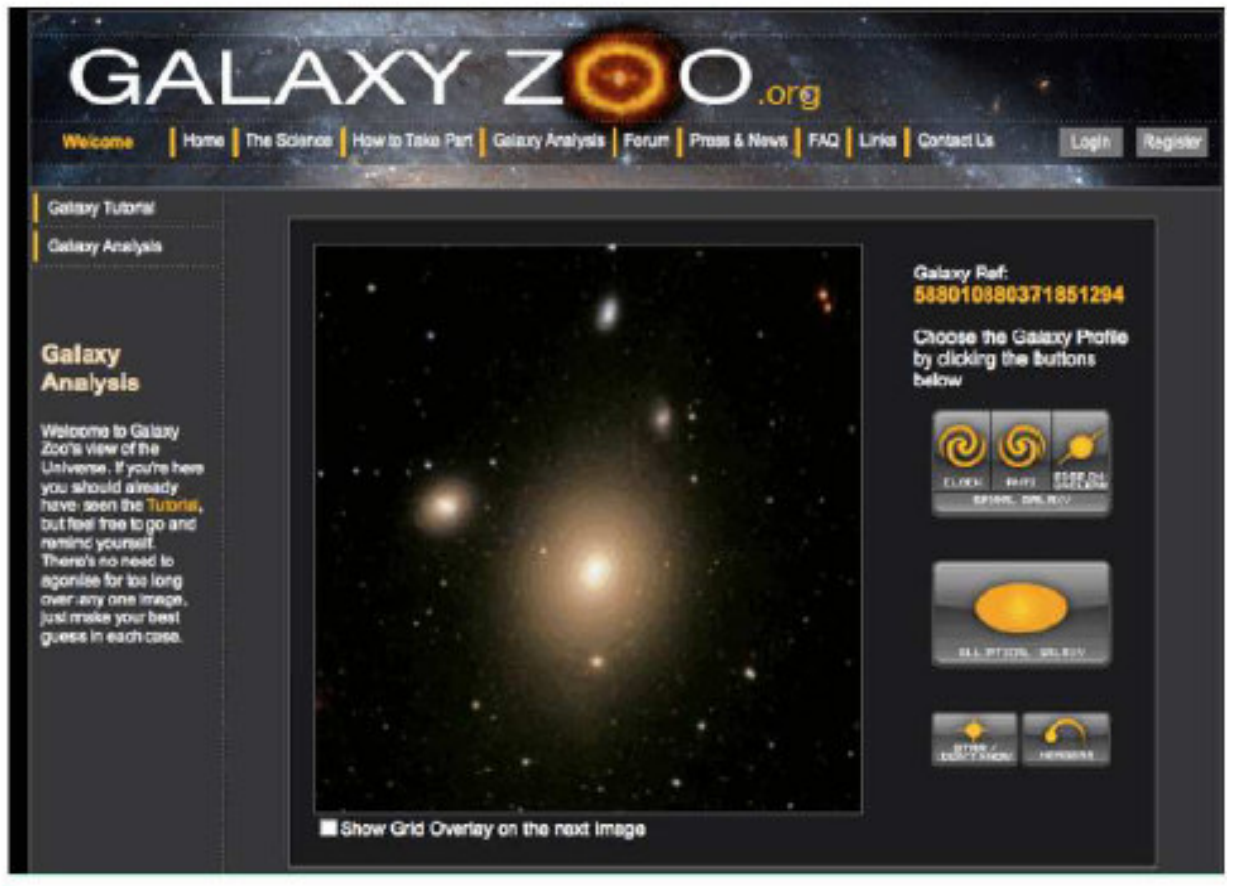

Fig. 2 The only question of Galaxyzoo1 menu

2) Galaxyzoo 2 . Analysed database was the same but the classification was much more detailed. An example of one of questions is the following 


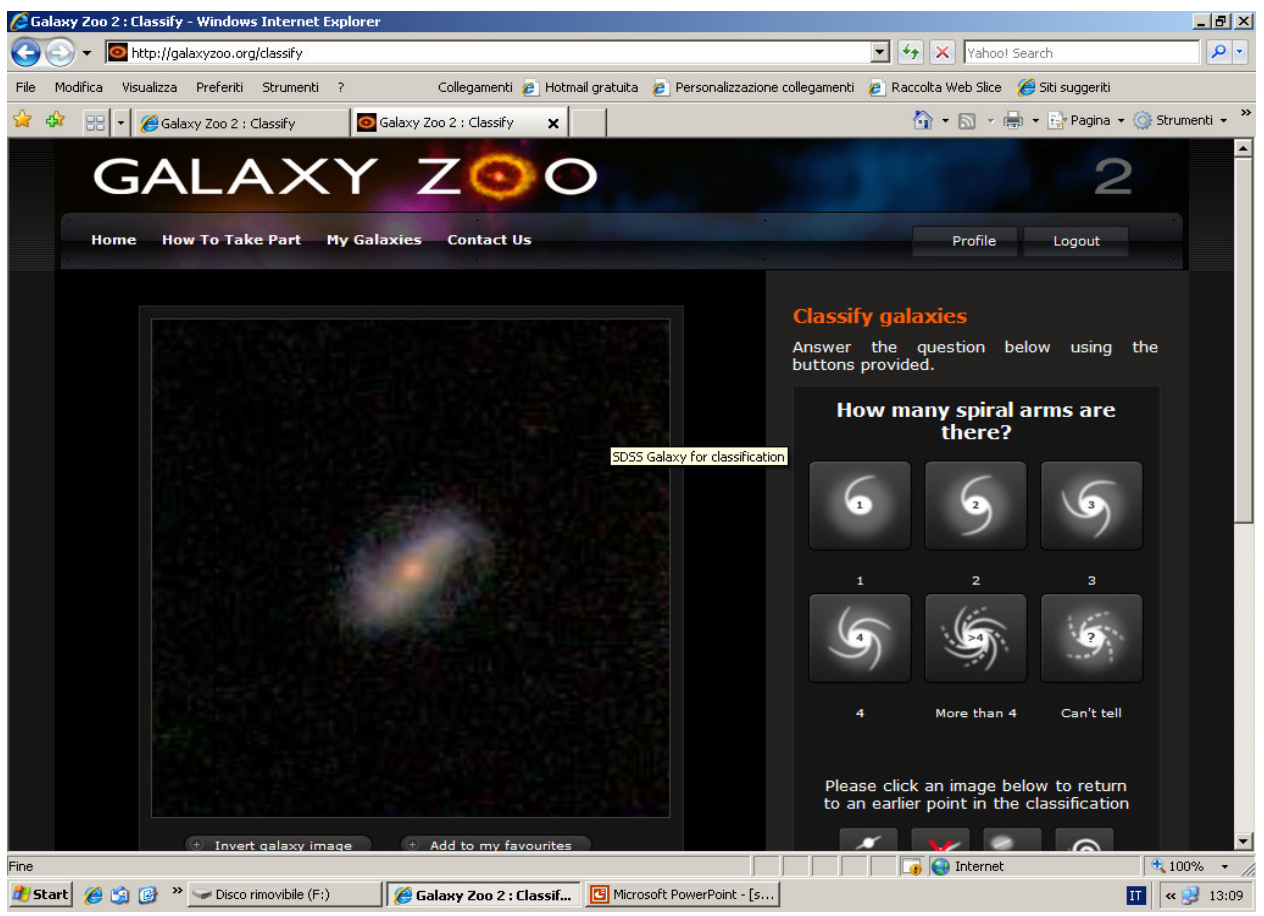

Fig. 3 An example of one of questions of Galaxyzoo2 menu

3) Actual stage is Galaxyzoo Hubble . It's to note that this stage wasn't till started when author presented Abstract of this communication. In Galaxyzoo Hubble there is the same detailed classification of Galaxyzoo 2 but on middle-high redshift galaxies photographed by Hubble telescope .

\section{Galaxyzoo and dark matter}

Galaxyzoo "sees " only the baryonic and bright part of Universe . But it sees this part in very accurate manner because its morphological analysis is very detailed. It can assert that $37 \%$ of galaxies are spiral galaxies and of these $25 \%$ have one arm, $38 \%$ have two arms , $13 \%$ have three arms and so on ( numbers aren't harbinger of real results, they are only examples ). And more sophisticated analysises can be made and have been made. It can be searched for a relation between number of arms in spiral galaxies and another feature of these galaxies ( mass , position in a cluster, presence of AGN , colour .... ) . Practically Galaxyzoo can built a very accurate " identity card " of Universe . It's true that Galaxyzoo galaxies aren't every galaxy of universe but they are a very large and very representative sample of universe .

It's known, from many years, that baryonic part is only a little part of the Universe and its features are deeply affected by " darks ", dark matter and dark energy. Computer simulations building virtual universes ( $\Lambda \mathrm{CDM}$ model ) there are and obviously these simulations start with some assumptions on " darks " features i.e. mass of particle constituing dark matter or value of dark enrgy density. In very recent times it has been discovered ${ }^{9}$ that every element of Hubble sequence of Galaxies there is in these virtual universes. Now the morphological analysis of 
Galaxyzoo can be made on a virtual universe too . and the results of these analysis on real and virtual Universes can be compared ..

Practically we have a identy card both of real Universe and of virtual universe .

But details of latter will depend from starting assumptions on " darks " and then , changing these starting hypotesis it's possible to see what values of these features reproduce results of real Universe .

At this point three observations are to be made

First : By this way I can study not only dark matter but dark energy too. The almost totality of the methods to study dark matter, don't investigate dark energy .

Second : It doesn't need to build accelerators as LHC or big expensive detectors . It needs only supercomputers to do simulations but these apparatus can be utilised for other studies too .

Third : A link is possible between analysis proposed in this paper and other experiments . For example let suppose a dark matter candidate particle is discovered by an AMS-like ${ }^{10}$ experiment or by one of LHC experiemnts at CERN and let admit its mass is 1.4 TEV . It's possible to insert this data in a Universe computer simulation and to see if it produces a virtual universe very similar to real ( as seen from Galaxyzoo data ). Vice versa is possible too .

\section{Conclusion}

At the end some final remarks .

It's perhaps the first time in the history of science that a scientific project in which the role of simple people ( not professional researchers ) is essential, can give an important contribution to a resolution of problems being to the boundary of human knowledge .

This is only a proposal of one of Zooties ( so amateur scientists of Galaxyzoo are called ). In the future times author wants to contact both the astronomers of Galaxyzoo and other scholars involved in computer simulations or in other " darks " researches so that this proposal can be realised . .

Last but not least the Galaxyzoo scheme can be reproduced in other fields of science . Let think to a big particles physics experiment producing an huge quantity of data. If these can be analysed by a PC based software ( PC version of Geant 4 or similar ) it would be possible to put these data in a database linked to an Internet site such a way an online community could study them .

\section{References}

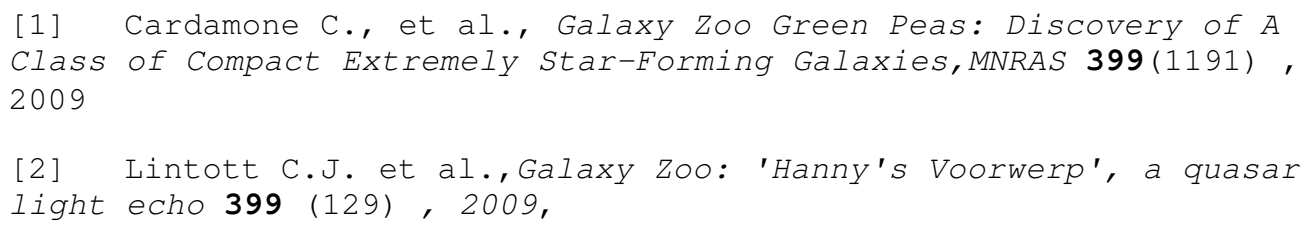


[3] Schawinski K., et al., Galaxy Zoo : A sample of blue early-type galaxies at low redshift MNRAS 396(818), 2009

[4] Masters K., et al., Galaxy Zoo: passive red spirals, MNRAS 405 $(783), 2010$

[5] Slosar A., et al.,Galaxy Zoo: Chiral correlation function of galaxy spins, MNRAS 392 (1225), 2008

[6] Bamford S.P., et al.,Galaxy Zoo: the dependence of morphology and colour on environment, MNRAS 393 (1324)

[7] Land K., et al., Galaxy Zoo: The large-scale spin statistics of spiral galaxies in the Sloan Digital Sky Survey, MNRAS, 388 (1686

[8] Schawinski K., et al. Galaxy Zoo: The Fundamentally Different Co-Evolution of Supermassive Black Holes and Their Early- and Late-Type Host Galaxies, ApJ 711 (284.)

[9] http://www.universetoday.com/2010/01/12/dark-energy-modelexplains-hubble-sequence-of-galaxies

[10] Kounine A., Status of AMS experiment , http://arxiv.org/ftp/arxiv/papers/1009/1009.5349.pdf 